\title{
Cirurgias bariátricas realizadas no Sistema Único de Saúde brasileiro entre 2010 e
}

\section{9}

\author{
Bariatric surgeries performed in the unique brazilian health system between 2010 and 2019
}

Cirugías bariátricas realizadas en el único sistema de salud brasileño entre 2010 y 2019

Recebido: 14/01/2021 | Revisado: 18/01/2021 | Aceito: 20/01/2021 | Publicado: 24/01/2021

\author{
Ananda Medeiros de Oliveira \\ ORCID: https://orcid.org/0000-0002-7688-5410 \\ Universidade Federal do Maranhão, Brasil \\ E-mail: anandamedeirosoliveira@hotmail.com \\ Sueli de Souza Costa \\ ORCID: https://orcid.org/0000-0003-4127-7324 \\ Universidade Federal do Maranhão, Brasil \\ E-mail: scsueli@gmail.com \\ Izolda Souza Costa \\ ORCID: https://orcid.org/0000-0003-0899-6845 \\ Universidade Federal do Maranhão, Brasil \\ E-mail: izolda.costa@hotmail.com \\ Nilson de Jesus Pereira Batalha Júnior \\ ORCID: https://orcid.org/0000-0002-6325-5655 \\ Universidade Federal do Maranhão, Brasil \\ E-mail: nilsonbatalha@gmail.com
}

\begin{abstract}
Resumo
A cirurgia bariátrica é um procedimento alternativo para controle da obesidade. Foi incluído no Sistema Único de Saúde em 1999 e, depois de vinte anos, ainda não supre a demanda de atendimento que cresce com as mudanças de hábito de vida da população. O presente estudo teve como objetivo levantar o número de bariátricas realizadas pelo sistema público brasileiro entre 2010 e 2019, quantificar os procedimentos por região, custo, complicações e taxa de mortalidade; além de promover visão crítica na academia. Através de dados secundários do Sistema de Informações Hospitalares do Sistema Único de Saúde, foi realizada análise quantitativa e descritiva das cirurgias bariátricas dentro das variáveis objetivadas, utilizando software estatístico para testes qui-quadrado de independência e regressão logística. Em dez anos, foram realizadas 79.961 cirurgias e investido $\mathrm{R} \$ 49.186$ milhões em bariátricas. A distribuição das cirurgias nas regiões brasileiras não foi igualitária, sendo o sul e sudeste os locais que mais realizaram procedimentos e receberam investimentos. O crescimento das cirurgias não demonstrou diferença estatística para $o$ número de óbitos, mas evidenciou concomitância nas complicações clínicas e cirúrgicas, com aumento de 266,66\% e $800 \%$, respectivamente. A técnica cirúrgica mais executada foi a gastroplastia com derivação intestinal (94,15\%), variando $4,7 \%$ dentro do observado. Apesar do quantitativo de cirurgias praticamente ter triplicado dentro do período avaliado, constata-se insuficiência para suprir a demanda gerada pela epidemia de obesidade, sendo necessário equidade dos investimentos nesse setor. Assim, percebe-se urgência do combate legítimo da problemática no país, com políticas públicas e educação em saúde efetivas.
\end{abstract}

Palavras-chave: Cirurgia bariátrica; Obesidade; Saúde pública.

\begin{abstract}
Bariatric surgery is an alternative procedure to control obesity. It was included in the Unified Health System in 1999 and, even after twenty years, it does not meet the growing demand for care who accompanies the population's changes in lifestyle. The present study aims to survey the number of bariatric patients performed by the Brazilian public system between 2010 and 2019, quantify the procedures by region, cost, complications and mortality rate; besides promoting critical vision in the academy. Using secondary data from the Hospital Information System of the Unified Health System, a quantitative and descriptive analysis of bariatric surgeries was performed within the targeted variables, analyzed through chi-square tests of independence and logistic regression. In ten years, there were 79,961 surgeries and invested $\mathrm{R} \$ 49,186$ million in bariatric. The distribution of surgeries in the Brazilian regions was not equal, with the south and southeast being the places that most performed procedures and received investments. The growth of surgery showed no statistical difference in the number of deaths, but the concomitant evident clinical and surgical complications with an increase of $266.66 \%$ and $800 \%$, respectively. The most performed surgical technique was gastroplasty with intestinal bypass (94.15\%), ranging within $4.7 \%$ observed. Although the number of surgeries has almost tripled within the period evaluated, it was not sufficient to supply the demand generated by the obesity
\end{abstract}


epidemic, requiring equity in investments in this sector. Thus, there is an urgency to legitimate combat of this problem in Brasil, with effective public policies and health education.

Keywords: Bariatric surgery; Obesity; Public health.

\section{Resumen}

Cirugía bariátrica es un procedimiento alternativo para controlar la obesidad. Ingresó al Sistema Único de Salud en 1999 y, después de veinte años, aún no satisface la demanda que crece con los cambios en el estilo de vida de la población. El presente estudio tiene como objetivo relevar el número de pacientes bariátricos realizados por el sistema público brasileño entre 2010-2019, para cuantificar los procedimientos por región, costo, complicaciones y tasa de mortalidad; además de promover visión crítica en la academia. Utilizando datos del Sistema de Información Hospitalaria, se realizó análisis cuantitativo y descriptivo de las cirugías bariátricas dentro de las variables objetivo, utilizando software estadístico para pruebas chi-cuadrado de independencia y regresión logística. En diez años se realizaron 79.961 cirugías y se invirtieron $\mathrm{R} \$ 49.186$ millones en bariatría. La distribución de las cirugías en las regiones brasileñas no fue igual, siendo el sur y el sureste los lugares que más procedimientos realizaron y recibieron inversiones. El crecimiento de las cirugías no mostró diferencia para el número de muertes, pero sí mostró concomitancia en las complicaciones clínicas y quirúrgicas, con un aumento del $266,66 \%$ y $800 \%$, respectivamente. La técnica quirúrgica más realizada fue gastroplastia con bypass intestinal $(94,15 \%)$, variando $4,7 \%$ dentro de lo observado. Aunque el número de cirugías prácticamente se ha triplicado, existe una insuficiencia para suplir la demanda generada por la obesidad, lo que requiere equidad en las inversiones. Por eso, es urgente el combate al problema en el país, con políticas públicas eficaces y educación en salud.

Palabras clave: Cirugía bariátrica; Obesidad; Salud pública.

\section{Introdução}

Cirurgias bariátricas são procedimentos realizados com intuito de reduzir a obesidade e auxiliar no controle de peso do indivíduo ao longo do tempo (Marcelino \& Patrício, 2011; Ramos \& Mello, 2015). São indicadas para pessoas que possuem obesidade grave ou obesidade moderada com uma doença associada, como: diabetes, hipertensão arterial ou apneia do sono tempo (Marcelino \& Patrício, 2011; ABESO, 2016; Zilberstein, Santo, \& Carvalho, 2019). Dados da Organização Mundial da Saúde relatam que aproximadamente $40 \%$ da polução mundial se encontra em sobrepeso ou com grau de obesidade (OMS, 2020), sendo o Brasil um dos dez países com maior prevalência de excesso de peso corporal (Wagner, Bastos, Navarro, Gonzalez-Chiva, \& Boing, 2018; Silva \& Branco, 2019); fato que reflete a crescente procura pelos procedimentos de gastroplastia (SBCBM, 2020a).

Essas cirurgias objetivam reduzir a capacidade do estômago de suportar a quantidade habitual de alimentos, trazendo sensação de saciedade, redução do consumo e emagrecimento (Marcelino \& Patrício, 2011; Ramos \& Mello, 2015). A maioria dos pacientes submetidos aos procedimentos relatam significativa melhora na qualidade de vida devido a produção de uma nova identidade corporal com a perda de peso, contribuindo também com aspectos de autoestima e interações sociais (Moreno, Silva, Bartholomeu, \& Montiel, 2011; Oliveira, Mazini, Venturini, Castro, \& Ferreira, 2018).

Apesar dos benefícios relatados frente a qualidade de vida dos indivíduos, alguns pontos negativos também são abordados na clínica, como má absorção de nutrientes e vitaminas, problemas gastrointestinais, transferência da compulsão alimentar para outro problema, entre outros (Gregorio et al., 2016; Oliveira et al., 2018; Vieira, 2018). Devido a isto, alguns critérios e diretrizes recomendados pelos órgãos e associações de saúde precisam ser seguidos para efetivação do procedimento (ABESO, 2016; CFM, 2020a; CFM, 2020b), além do acompanhamento de equipe multidisciplinar após a realização da operação (Ramos \& Mello, 2015; Miranda et al., 2019).

O Sistema Único de Saúde (SUS) brasileiro só incluiu a gastroplastia no rol de cobertura de procedimentos nove anos depois da lei que implementa o sistema (Carvalho \& Rosa, 2018) e, até hoje, não consegue aportar a demanda que cresce consideravelmente a cada ano, sendo relatado que aproximadamente cinco milhões de brasileiros se enquadram nos critérios estabelecidos para realização da cirurgia bariátrica (Bhering et al., 2018). Em virtude dessa demanda, parte dos procedimentos realizados são buscados no setor de atendimento privado a saúde e se constitui um ônus para o indivíduo, marginalizando pessoas que também necessitam de assistência e, por vezes, não conseguem solução (Kelles, Machado, \& Barreto, 2014; 
SBCBM, 2020a).

Desta forma, este estudo visou analisar a evolução do número de cirurgias bariátricas realizadas no SUS entre os anos de 2010 e 2019, determinar região do país que mais realiza o procedimento, definir qual o tipo de técnica para redução de estômago é mais empregada, taxa de mortalidade, intercorrências médicas e estimar os custos da cirurgia bariátrica para a saúde pública; além de fomentar o pensamento crítico da academia para necessidade de políticas públicas mais efetivas para essa demanda.

\section{Metodologia}

\subsection{Tipo de estudo, fonte de dados e variáveis abordadas}

Trata-se de um estudo de cunho ecológico, de série temporal, quali-quantitativo, baseado em dados secundários sobre o número de cirurgias bariátricas (Pereira, Shitsuka, Parreira, \& Shitsuka, 2018). A fonte dos dados utilizada foi de arquivos públicos do Sistema de Informações Hospitalares do SUS (SIH/SUS), correspondentes ao período do ano de 2010 a 2019 no Departamento de Informática do SUS (DATASUS), do Ministério da Saúde do Brasil. As variáveis utilizadas foram ano, região, tipo de cirurgia, taxa de mortalidade e intercorrências clínicas.

\subsection{Análise estatística}

A análise dos dados foi realizada com o software IBM® SPSS® Statistics 20 (2011). A avaliação da associação do cruzamento das variáveis classificatórias (ano, região, tipo de cirurgia, taxa de mortalidade e intercorrências clínicas) foi feita através do teste qui-quadrado $\left(\chi^{2}\right)$ de independência. A avaliação da evolução da taxa de mortalidade em relação ao ano foi feita pela regressão logística. Em todos os testes, o nível de significância $(\alpha)$ utilizado foi de $5 \%$, ou seja, foi considerado significativo quando $\mathrm{p}<0,05$ (Callegari-Jacques, 2009).

\subsection{Aspectos éticos}

Por envolver apenas dados secundários e de domínio público, este estudo não necessitou de aprovação por parte do Comitê de Ética em Pesquisa, fundamentando-se nas diretrizes e normas regulamentadoras estabelecidas nas Resoluções $n^{\circ}$ 466/2012 e 580/2018 do Conselho Nacional de Saúde do Ministério da Saúde.

\section{Resultados}

\subsection{Cirurgias bariátricas realizadas e sua distribuição por região do país}

A distribuição do número de cirurgias bariátricas em relação ao ano e as regiões brasileiras se encontram na Tabela 1. Observa-se que no ano de 2010 foram realizados 4.441 procedimentos de gastroplastias, enquanto no ano de 2019 esse número foi 12.541 cirurgias, evidenciando crescimento de 180,05\% em relação à média do período de estudo avaliado. A região sul foi a que mais realizou procedimentos dentro do período analisado (58,55\%), sendo a região sudeste a segunda em classificação $(32,63 \%)$. Nota-se que nas demais regiões do país (norte, nordeste e centro-oeste) a proporção do número de cirurgias não mudou muito ao longo dos anos. Foi possível, também, identificar variação na proporção das cirurgias por região e ano, com associação significativa entre elas $\left(\chi^{2}=6225,03 ; \mathrm{p}<0,05\right)$. 
Tabela 1. Distribuição do número de cirurgias em relação ao ano e as regiões brasileiras no período de 2010 a 2019.

\begin{tabular}{cccccccccccc}
\hline & \multicolumn{1}{c}{ Regiões brasileiras } & \multicolumn{1}{c}{} \\
\cline { 2 - 9 } & norte & \% & nordeste & \% & sudeste & \% & sul & \% & centro-oeste & \% & Total \\
\hline 2010 & 40 & 0,90 & 413 & 9,30 & 1.491 & 33,57 & 2.343 & 52,76 & 154 & 3,57 & 4.441 \\
2011 & 62 & 1,18 & 487 & 9,31 & 1.841 & 35,22 & 2.729 & 52,21 & 108 & 2,07 & 5.227 \\
2012 & 91 & 1,51 & 581 & 9,68 & 2.102 & 35,00 & 3.149 & 52,44 & 82 & 1,37 & 6.005 \\
2013 & 97 & 1,43 & 534 & 7,89 & 2.405 & 35,52 & 3.636 & 53,70 & 99 & 1,46 & 6.771 \\
2014 & 61 & 0,87 & 483 & 6,90 & 2.628 & 37,53 & 3.709 & 52,96 & 122 & 1,74 & 7.003 \\
2015 & 101 & 1,35 & 487 & 6,49 & 2.764 & 36,85 & 3.998 & 53,30 & 151 & 2,01 & 7.501 \\
2016 & 65 & 0,73 & 593 & 6,74 & 3.196 & 36,31 & 4.906 & 55,74 & 42 & 0,48 & 8.802 \\
2017 & 38 & 0,37 & 537 & 5,22 & 3.146 & 30,58 & 6.565 & 63,82 & 1 & 0,01 & 10.287 \\
2018 & 31 & 0,27 & 531 & 4,66 & 3.238 & 28,45 & 7.529 & 66,14 & 54 & 0,47 & 11.383 \\
2019 & 42 & 0,33 & 591 & 4,71 & 3.278 & 26,14 & 8.256 & 65,83 & 374 & 2,98 & 12.541 \\
\hline
\end{tabular}

Teste

$\chi^{2}=6225,03 \mathrm{p}<0,0001$

Fonte: DATASUS.

\subsection{Custo das cirurgias por ano e região do Brasil}

Sobre o custo das cirurgias de gastroplastias, a Tabela 2 permite a visualização dos gastos frente ao ano e região do país. Os maiores investimentos no sistema de saúde para o procedimento avaliado se deram na região sul e sudeste, com demanda acumulada dentro do tempo analisado de $60 \%$ e $32 \%$ do valor dispensado.

Tabela 2. Gastos em reais com cirurgias nas regiões do país, no período de 2010 a 2019.

\begin{tabular}{ccccccc}
\hline \multirow{2}{*}{ Ano } & \multicolumn{3}{c}{ Regiões brasileiras } & \multicolumn{2}{c}{ Total } \\
\cline { 2 - 6 } & norte & nordeste & sudeste & sul & centro-oeste & 245,72 \\
2010 & 2,29 & 22,93 & 82,47 & 129,68 & 8,35 & 283,89 \\
2011 & 3,55 & 26,04 & 98,30 & 150,30 & 5,69 & 323,95 \\
2012 & 5,03 & 32,30 & 111,72 & 171,42 & 3,43 & 416,58 \\
2013 & 6,31 & 31,65 & 147,56 & 224,95 & 6,11 & 444,88 \\
2014 & 3,21 & 31,40 & 160,78 & 241,47 & 8,01 & 469,26 \\
2015 & 6,42 & 29,15 & 174,63 & 250,88 & 8,18 & 563,40 \\
2016 & 3,60 & 37,14 & 197,99 & 322,23 & 2,44 & 657,84 \\
2017 & 2,47 & 34,15 & 194,48 & 426,67 & 0,06 & 711,57 \\
2018 & 1,79 & 32,10 & 191,02 & 483,26 & 3,41 & 801,60 \\
2019 & 2,53 & 37,14 & 202,64 & 534,66 & 24,59 & 70,27 \\
\hline Total & 37,20 & 314,00 & $1.561,60$ & $2.935,53$ & & 4,60 \\
\hline
\end{tabular}

Legenda: Valores expressos em reais (R\$), moeda brasileira, multiplicados por 100 mil. Fonte: DATASUS

\subsection{Taxa de mortalidade}

De acordo com a Tabela 3, não foi encontrado uma diferença significativa no número de óbitos em relação ao ano, no período do estudo $\left(\chi^{2}=6,54 ; p>0,05\right)$, havendo redução da taxa de mortalidade de 0,31 para 0,15 entre os anos de 2010 e 2019 . 
Tabela 3. Número de óbitos e taxa de mortalidade das cirurgias bariátricas por ano no período de 2010 a 2019.

\begin{tabular}{lccc}
\hline Ano & Número de óbitos & Taxa da mortalidade & $\chi^{2}$ \\
\hline 2010 & 14 & 0,31 \\
2011 & 11 & 0,21 \\
2012 & 11 & 0,18 \\
2013 & 15 & 0,22 & 6,54 \\
2014 & 13 & 0,19 & 0,6849 \\
2015 & 10 & 0,13 & \\
2016 & 19 & 0,22 & \\
2017 & 12 & 0,12 & \\
2018 & 15 & 0,13 \\
2019 & 19 & 0,15 \\
\hline
\end{tabular}

\section{Fonte: DATASUS}

\subsection{Intercorrências clínicas e cirúrgicas em gastroplastias}

O Sistema de Informações Hospitalares do SUS não possuía dados compilados sobre o número de intercorrências das cirurgias bariátricas entre os anos de 2010 e 2012, forçando esta análise inferir apenas sobre os anos posteriores avaliados.

A proporção de intercorrências clínicas das cirurgias variou significativamente de acordo com as regiões $\left(\chi^{2}=108.75\right.$; p < 0,05), sendo maior na região sul (Tabela 4). Por sua vez, as intercorrências cirúrgicas também demonstraram variação pertinente com o aumento do número de cirurgias, sendo sua frequência absoluta menor que as complicações clínicas (Tabela 5). As regiões norte e centro-oeste apresentaram o menor índice de complicações na cirurgia, mas também são as regiões do país que menos realizam esse tipo de cirurgia (Tabelas 4 e 5).

Tabela 4. Distribuição do número de intercorrências clínicas das cirurgias bariátricas por ano em relação a região do Brasil.

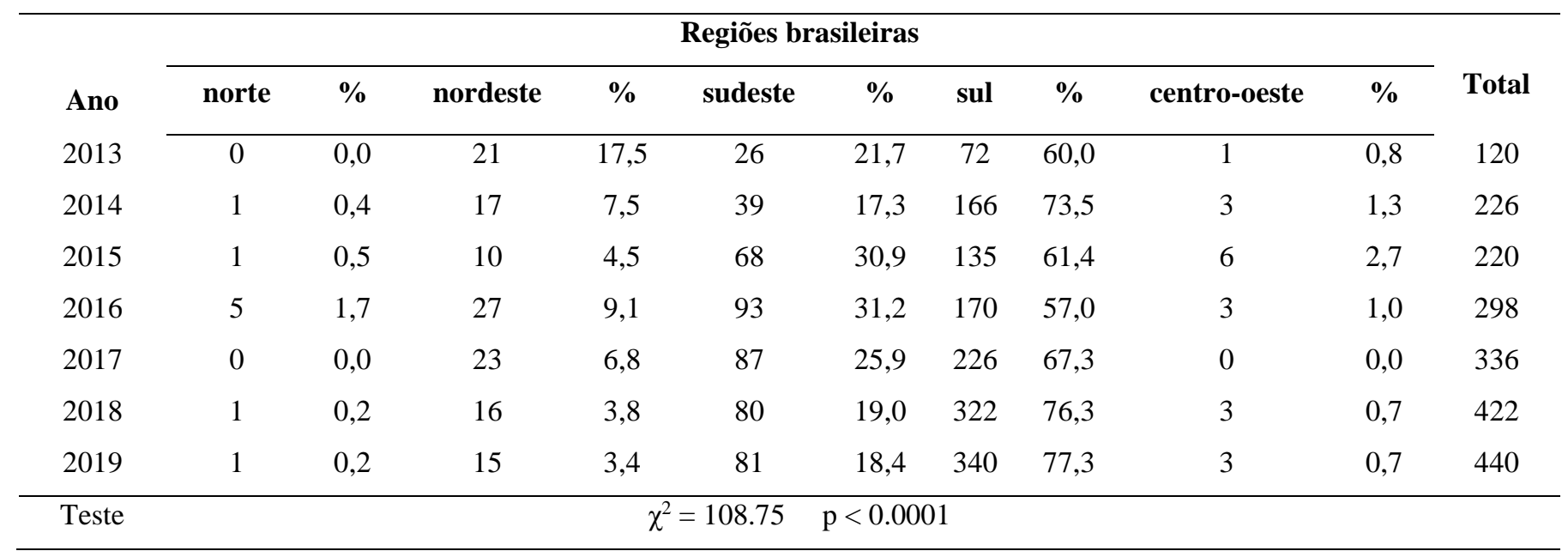


Tabela 5. Distribuição do número de intercorrências cirúrgicas das gastroplastias por ano em relação a região do Brasil.

\begin{tabular}{|c|c|c|c|c|c|c|c|c|c|c|c|}
\hline \multirow[b]{2}{*}{ Ano } & \multicolumn{9}{|c|}{ Regiões brasileiras } & \multirow[b]{2}{*}{$\%$} & \multirow[b]{2}{*}{ Total } \\
\hline & norte & $\%$ & nordeste & $\%$ & sudeste & $\%$ & sul & $\%$ & centro-oeste & & \\
\hline 2013 & 0 & 0,00 & 0 & 0,00 & 2 & 11,1 & 14 & 77,8 & 2 & 11,1 & 18 \\
\hline 2014 & 1 & 1,9 & 2 & 3,8 & 7 & 13,5 & 42 & 80,8 & 0 & 0.00 & 52 \\
\hline 2015 & 0 & 0,00 & 5 & 5,7 & 36 & 40,9 & 46 & 52,3 & 1 & 1,1 & 88 \\
\hline 2016 & 0 & 0,00 & 8 & 11,4 & 14 & 20,0 & 47 & 67,1 & 1 & 1,5 & 70 \\
\hline 2017 & 0 & 0,00 & 11 & 10,6 & 19 & 18,3 & 74 & 71,1 & 0 & 0,00 & 104 \\
\hline 2018 & 0 & 0,00 & 15 & 11,3 & 14 & 10,7 & 103 & 78,0 & 0 & 0,00 & 132 \\
\hline 2019 & 0 & 0,00 & 5 & 3,1 & 22 & 13,6 & 135 & 83,3 & 0 & 0,00 & 162 \\
\hline
\end{tabular}

Fonte: DATASUS

\subsection{Tipos de gastroplastias}

Dentre os tipos de técnicas cirúrgicas, a gastroplastia com derivação intestinal foi a mais frequente em todos os anos, variando de 91,5\% a 96,2\% (Tabela 6). A frequência de cirurgias realizadas pelo método de gastrectomia vertical em manga (sleeve) foi nula entre os anos de 2010 e 2012 e por videolaparoscopia nula entre 2010 a 2016. Assim, a fórmula de obtenção de $\chi^{2}$ produziu um valor significativo (> do que o $\chi^{2}$ crítico), sendo necessário usar um fator de correção de continuidade para cada classe. Não foi encontrado nesse estudo uma associação significativa na proporção do tipo de cirurgia bariátrica em relação aos anos $\left(\chi^{2}\right.$ com correção de Yates $\left.=3.57 ; p>0,05\right)$.

Tabela 6. Distribuição do tipo de cirurgia bariátrica por ano.

\begin{tabular}{lccccccccc}
\hline Ano & GDI & \% & GVB & \% & CBV & \% & GVM & \% & Total \\
\hline 2010 & 4251 & 95.7 & 190 & 4.3 & 0 & 0.0 & 0 & 0.0 & 4441 \\
2011 & 5026 & 96.2 & 201 & 3.8 & 0 & 0.0 & 0 & 0.0 & 5227 \\
2012 & 5748 & 95.7 & 257 & 4.3 & 0 & 0.0 & 0 & 0.0 & 6005 \\
2013 & 6471 & 95.6 & 152 & 2.2 & 0 & 0.0 & 148 & 2.2 & 6771 \\
2014 & 6592 & 94.1 & 71 & 1.0 & 0 & 0.0 & 340 & 4.9 & 7003 \\
2015 & 6883 & 91.8 & 69 & 0.9 & 0 & 0.0 & 549 & 7.3 & 7501 \\
2016 & 8423 & 95.7 & 64 & 0.7 & 0 & 0.0 & 315 & 3.6 & 8802 \\
2017 & 9803 & 95.3 & 49 & 0.5 & 218 & 2.1 & 217 & 2.1 & 10287 \\
2018 & 10610 & 93.2 & 26 & 0.2 & 539 & 4.7 & 208 & 1.8 & 11383 \\
2019 & 11478 & 91.5 & 22 & 0.2 & 235 & 1.9 & 806 & 6.4 & 12541 \\
\hline Teste & & & $\chi^{2}$ com correção de Yates $=3.57$ & $\mathrm{p}=0.9997$ & & &
\end{tabular}

Legenda: GDI = gastroplastia com derivação intestinal; GVB = gastroplastia vertical com banda; CBV = cirurgia bariátrica por videolaparoscopia; GVM = gastrectomia vertical em manga. Fonte: DATASUS

\section{Discussão}

Ao analisar os resultados do estudo, evidencia-se aumento significativo no número de cirurgias bariátricas realizadas 
anualmente pelo SUS no Brasil, tendo esse número praticamente triplicado entre os anos de 2010 e 2019. Realidade que se correlaciona com o aumento concomitante do número de obesos no país (Wagner et al., 2018; Silva \& Branco, 2019) e se ratifica em pesquisa realizada pela Vigilância de Fatores de Risco e Proteção para Doenças Crônicas por Inquérito Telefônico (VIGITEL), que apontou crescimento aproximado de 53\% na prevalência da doença nos dez anos avaliados (Brasil, 2017).

Esse crescimento da prevalência é visualizado em todas as regiões do país (Malta, Silva, Tonaco, Freitas \& Velasquez-Melendez, 2019). Mas quando se observa o atendimento cirúrgico dispensado à população que busca o SUS, discrepâncias em relação ao quantitativo de procedimentos realizados por região se apresentam. Tonatto-Filho, Galloti, Chedid, Grezzana-Filho e Garcia (2019) também evidenciaram que as localidades mais desenvolvidas do Brasil, como sul e sudeste, disponibilizam maior oferta pública desse procedimento cirúrgico e atribuem essa demanda a relação de mais profissionais especializados em cirurgias digestivas nesses centros econômicos do país frente a outras regiões.

Para se estimar os custos de uma doença para o sistema público, analisa-se variáveis como gastos diretos, indiretos e intangíveis. No caso da obesidade, como se associa a outros problemas, o diagnóstico principal nem sempre acontece, dificultando uma real estimativa do que é dispensado para problemática (Bahia \& Araújo, 2014). Pesquisa brasileira recente estimou gastos totais para hipertensão, diabetes e obesidade no SUS alcançando somas de 3,45 bilhões de reais e reafirmou a dificuldade na separação das doenças, visto que a obesidade tem etiologia multifatorial (Nilson, Andrade, Brito, \& Oliveira, 2018).

Depois de se tentar todos os métodos não invasivos para perda de peso, a cirurgia se apresenta como recurso mais efetivo de tratamento (Ramos \& Mello, 2015; Kelles et al., 2018; Elias et al., 2018). Nos últimos dez anos, o SUS investiu R\$ 49.186 milhões em gastroplastias e ofertou 79.961 cirurgias. O montante aplicado pelo sistema, ao ser analisado por um único prisma parece elevado, mas os dispêndios à saúde pública com a realização das cirurgias são benéficos e contribuem para diminuição de outros gastos públicos oriundos de doenças secundárias ocasionadas pela obesidade (Zumbiaurre et al., 2017).

Atualmente, algumas técnicas são usadas pelos cirurgiões do aparelho digestivo para concretização da bariátrica, como gastroplastia com derivação intestinal, gastroplastia vertical com banda, cirurgia bariátrica por videolaparoscopia e gastrectomia vertical em manga ou sleeve (Tonnato-Filho et al., 2019). As cirurgias também se diferem quanto aos mecanismos de funcionamento, podendo ser restritivas, disabsortivas e mistas (Ramos \& Mello, 2015). No SUS, evidencia-se variação da frequência de realização de cada uma das técnicas cirúrgicas, sendo a gastroplastia com derivação intestinal a mais empregada; a literatura também corrobora com esse achado (Tonnato-Filho et al., 2019).

Alguns métodos cirúrgicos para execução da bariátrica começaram a ser realizados pelo SUS depois do período de início estipulado por esta análise, demandando estatística diferenciada para correlação. Esse fato se alinha com dados da Sociedade Brasileira de Cirurgia Bariátrica e Metabólica (SBCBM, 2020b) que remontam preferência por algumas técnicas e abandono de outras, como a gastroplastia vertical com banda devido à necessidade do uso de um anel de silicone inflável, que pode trabalhar como um corpo estranho no organismo e gerar complicações no pós-operatório, mesmo depois de anos (Elias et al., 2011; SBCBM, 2020b).

A cirurgias realizada por meio de videolaparoscopia é mais um dos procedimentos que só foram introduzidos recentemente no sistema público de saúde. Devido a isto, o quantitativo registrado nas análises para este método é baixo, mesmo ele apresentando menor taxa de mortalidade, melhor recuperação e aspecto estético dentre todos os outros (Elias et al., 2011; SBCBM, 2020b). Essa técnica não está disponível em todos os hospitais especializados devido ao alto custo dos equipamentos médicos necessários para sua realização e suscita debate frente ao custo benefício, visto que mais investimentos nesse método podem reduzir problemas aos pacientes em decorrência da cirurgia, bem como favorecer menor gasto hospitalar com tempo de internação e manutenção do indivíduo dentro do estabelecimento de saúde (Elias et al., 2011).

Com o passar do tempo, assim como em qualquer outro procedimento médico, a ciência evolui, especializa e aprimora 
os protocolos, refletindo na resposta da intervenção e redução da taxa de mortalidade (Kelles et al., 2014; Penna, Vaz, Fonseca, Kalichsztein, \& Nobre, 2017), como observado também em nosso estudo. No entanto, ao contrastar os dados de taxa de mortalidade e complicações dos procedimentos, percebe-se que o aprimoramento das equipes não foi acompanhado no que tange às complicações clínicas e cirúrgicas, com aumento de 266,66\% e 800\%, respectivamente, entre os anos 2013 e 2019.

Teoriza-se que este fato pode ser explicado pela necessidade não só de uma equipe cirúrgica especializada e eficiente, mas do acompanhamento multidisciplinar do paciente com profissionais da saúde antes e depois da cirurgia com o intuito de reduzir as complicações. Estudos (Ramos \& Mello, 2015; Miranda et al., 2019) afirmam que os cuidados com os pacientes que realizam gastroplastias devem ser contínuos, caso contrário podem trazer riscos à efetividade do procedimento e à saúde do indivíduo submetido.

Mesmo com aumento do número de complicações, a frequência do aparecimento deste evento é pouco significativo comparado ao total de cirurgias realizadas ao ano. Vale salientar, ainda, que a cirurgia bariátrica quando sugerida e bem avaliada pelo médico, materializa-se como um procedimento seguro, com baixa probabilidade de complicações intraoperatórias e taxa de mortalidade (Kelles et al., 2014; Penna et al., 2017); além de favorecer melhor qualidade de vida ao indivíduo (Oliveira et al., 2018).

Apesar da constatação de crescente número de cirurgias e o implemento de técnicas com menos riscos realizadas pelo SUS, o quantitativo dos procedimentos ainda se apresenta como deficiente para a demanda, visto que muitas pessoas elegíveis para cirurgia podem ficar até quatro anos na fila de espera (Tonatto-Filho et al., 2019) e que a maior parte dos brasileiros depende exclusivamente do sistema público (Kelles et al., 2014).

Dados da SBCBM (2020a) alertam sobre essa problemática em levantamento, demonstrando que das 63.969 gastroplastias realizas em 2018, 82,17\% foram executadas pela rede privada de saúde. No entanto, para este mesmo ano, 13.600.000 pessoas eram elegíveis para realização do procedimento, sendo que apenas $0,47 \%$ destes realmente fizeram a cirurgia e que somente $0,08 \%$ foram pelo sistema público.

Nota-se a necessidade de ampliação do número de cirurgias ofertadas pelo SUS, com investimentos em hospitais, equipamentos para realização de procedimentos menos invasivos e capacitação de equipe multidisciplinar para diminuir as complicações. Além de tudo isso, implementar políticas públicas com foco na conscientização alimentar da população, acessibilidade de alimentos saudáveis, aumento de taxas e rotulagem diferenciada sobre produtos não saudáveis, semelhante ao observado em países como Chile e México (Martins, 2018; Malta et al., 2019). Outras ações incluem promoção de educação e políticas em saúde que, seguidas, podem contribuir para diminuição de novos casos de obesidade, redução de gastos secundários do sistema público e fomentar na população a ciência dos efeitos danosos da epidemia do excesso de peso.

\section{Conclusão}

O crescimento na realização de cirurgias bariátricas realizadas pelo SUS tem relação direta com o avanço da epidemia de obesidade no país, visto que o procedimento, depois da não assertiva de todos os outros tratamentos, apresenta-se como mais efetivo, seguro e com índices de complicações baixos. A distribuição regional do procedimento se materializa de maneira desigual e remonta a carência de investimentos em algumas localidades, associado ao fato preocupante de técnicas menos invasivas não serem as mais empregadas.

Em virtude da importância dos dados demonstrados, sugere-se que periodicamente novas pesquisas sobre a temática venham a público com intuito de atualização constante dos levantamentos, favorecendo difusão técnico-científica, ações de políticas públicas e, ainda, lançar base para gerenciamento equitativo dos investimentos em saúde de forma efetiva as regiões brasileiras. 


\section{Referências}

Associação Brasileira para o Estudo da Obesidade e da Síndrome Metabólica (ABESO). Diretrizes Brasileiras de Obesidade. (4a ed.), 2016.

Bahia, L., \& Araújo, D. V. (2014). Impacto econômico da obesidade no Brasil. Revista Hospital Universitário Pedro Ernesto, 13(1).

Bhering, N. B. V., Aquino, M. L. A., Thiede, A. N., Vilella, A. M. C., Dorneles, A. R., dos Santos Duque, A. C. F., Duque A. C. F. S., Vieira A. A. D., Lages BPG, \& Ferreira, N. L. C. (2020). Uso do ácido Ursodesoxicólico na prevenção da colelitíase em pacientes com bypass gástrico em Y de Roux: Uma revisão. Brazilian Journal of Health Review, 3(4), 10790-10801.

Brasil (2017). Ministério da Saúde. VIGITEL Brasil 2016: Hábitos dos brasileiros impactam no crescimento da obesidade e aumenta prevalência de diabetes e hipertensão. http://www.saude.gov.br

Callegari-Jacques, S. M. (2009). Bioestatística: princípios e aplicações. Artmed Editora.

Carvalho, A. D. S., \& Rosa, R. D. S. (2018). Cirurgias bariátricas realizadas pelo Sistema Único de Saúde em residentes da Região Metropolitana de Porto Alegre, Rio Grande do Sul, 2010-2016. Epidemiologia e Serviços de Saúde, 27, e2017010.

Conselho Federal de Medicina - CFM (2020a). CFM detalha lista de comorbidades que podem levar a indicação da cirurgia bariátrica. http://www.portal.cfm.org.br

Conselho Federal de Medicina - CFM (2020b). CFM divulga critérios exigidos para a realização de cirurgia metabólica no País. http://www.portal.cfm.org.br

Elias, A. A., Garrido-Junior, A. B., Berti, L. V., Oliveira, M. R. D., Bertin, N. T. S., Malheiros, C. A., \& Bastouly, M. (2011). Derivações gástricas em y-deroux com anel de silicone para o tratamento da obesidade: estudo das complicações relacionadas com o anel. Arquivos Brasileiros de Cirurgia Digestiva, 24(4), 290-295.

Elias, A. A., Roque-de-Oliveira, M., Campos, J. M., Sasake, W. T., Bandeira, Á. A., Silva, L. B., Ferreira B., Ito R. M., Shirozaki H. Y., Benetti F. A., Paiva L. S., \& Garrido Júnior, A. B. (2018). Cirurgia bariátrica robótico-assistida: análise de série de casos e comparação com via laparoscópica. Revista do Colégio Brasileiro de Cirurgiões, 45(3).

Gregorio, V. D., Lucchese, R., Vera, I., Silva, G. C., Silva, A., \& MORAES, R. C. C. (2016). The alcohol consumption is amended after bariatric surgery? An integrative review. Arquivos Brasileiros de Cirurgia Digestiva, 29, 111-115.

Kelles, S. M. B., Machado, C. J., \& Barreto, S. M. (2014). Dez anos de cirurgia bariátrica no Brasil: mortalidade intra-hospitalar em pacientes atendidos pelo Sistema Único de Saúde ou por Operadora da Saúde Suplementar. Arquivos Brasileiros de Cirurgia Digestiva, 27(4), 261-7.

Malta, D. C., Silva, A. G. D., Tonaco, L. A. B., Freitas, M. I. D. F., \& Velasquez-Melendez, G. (2019). Tendência temporal da prevalência de obesidade mórbida na população adulta brasileira entre os anos de 2006 e 2017. Cadernos de Saúde Pública, 35, e00223518.

Marcelino, L. F., \& Patrício, Z. M. (2011). A complexidade da obesidade e o processo de viver após a cirurgia bariátrica: uma questão de saúde coletiva. Ciência \& Saúde Coletiva, 16(12), 4767-4776.

Martins, A. P. B. (2018). É preciso tratar a obesidade como um problema de saúde pública. Revista de Administração de Empresas, 58(3), 337-341.

Miranda, R. C. D. D., Radünz, V., Sebold, L. F., Rosa, L. M. D., Girondi, J. B. R., \& Tourinho, F. S. V. (2019). Communication technologies of a nutrition service contributing to the safety of bariatric surgery patients. Texto \& Contexto-Enfermagem, 28.

Moreno, C. A. S., Silva, A. M., Cecato, J. F., Bartholomeu, D., \& Montiel, J. M. (2011). Caracterização das mudanças psicológicas ocasionadas em indivíduos submetidos à cirurgia bariátrica. Encontro: Revista de Psicologia, 14(20), 99-116.

Nilson, E. A. F., Andrade, R. D. C. S., Brito, D. A. D., \& Oliveira, M. L. D. (2020). Custos atribuíveis a obesidade, hipertensão e diabetes no Sistema Único de Saúde, Brasil, 2018. Revista Panamericana de Salud Pública, 44, e32.

Oliveira, L. S. F., Mazini Filho, M. L., Venturini, G., de Castro, J. B. P., \& Ferreira, M. E. C. (2018). Repercussões da cirurgia bariátrica na qualidade de vida de pacientes com obesidade: uma revisão integrativa. RBONE-Revista Brasileira De Obesidade, Nutrição E Emagrecimento, 12(69), $47-58$.

Organização Mundial da Saúde - OMS (2020). Obesity and overweight. http://www.who.int

Penna, G. L. D. A., Vaz, I. P., Fonseca, E. C., Kalichsztein, M., \& Nobre, G. F. (2017). Pós-operatório imediato de cirurgia bariátrica em unidade intensiva versus unidade de internação. Estudo retrospectivo com 828 pacientes. Revista Brasileira de Terapia Intensiva, 29(3), 325-330.

Pereira, A. S., Shitsuka, D. M., Parreira, F. J., \& Shitsuka, R. (2018). Metodologia da pesquisa científica. http://repositorio.ufsm.br/bitstream/handle/1 /15824/Lic_Computacao_Metodologia-Pesquisa-Cientifica.pdf?sequence=1

Ramos, C. P., \& de Mello, E. D. (2015). Manejo nutrológico no pós-operatório de cirurgia bariátrica. International Journal of Nutrology, 8(02), 039-049.

Silva, M. O. D., \& Branco, A. U. (2019). Obesity, Prejudice, Self, and Culture: A Longitudinal Case Study. Paidéia, 29.

Sociedade Brasileira de Cirurgia Bariátrica e Metabólica - SBCBM (2020a). Cirurgia bariátrica cresce 84,73\% entre 2011 e 2018. http://www.sbcbm.org.br

Sociedade Brasileira de Cirurgia Bariátrica e Metabólica - SBCBM (2020b). Cirurgia Bariátrica - Técnicas Cirúrgicas. http://www.sbcbm.org.br

Tonatto-Filho, A. J., Gallotti, F. M., Chedid, M. F., Grezzana-Filho, T. D. J. M., \& Garcia, A. M. S. V. (2019). Bariatric surgery in brazilian public health system: the good, the bad and the ugly, or a long way to go. yellow sign!. Arquivos Brasileiros de Cirurgia Digestiva, 32(4). 
Research, Society and Development, v. 10, n. 1, e47510111985, 2021

(CC BY 4.0) | ISSN 2525-3409 | DOI: http://dx.doi.org/10.33448/rsd-v10i1.11982

Vieira, E. (2018). Complicações decorrentes de cirurgia bariátrica em pacientes atendidos em um hospital no sul de Santa Catarina. Arquivos Catarinenses de Medicina, 47(3), 74-84.

Wagner, K. J. P., Bastos, J. L. D., Navarro, A., Gonzalez-Chica, D. A., \& Boing, A. F. (2018). Posição socioeconômica na infância e obesidade em adultos: estudo de base populacional. Revista de Saúde Pública, 52, 15.

Zilberstein, B., Santo, M. A., \& Carvalho, M. H. (2019). Critical analysis of surgical treatment techniques of morbid obesity. Arquivos Brasileiros de Cirurgia Digestiva, 32(3).

Zubiaurre, P. R., Bahia, L. R., da Rosa, M. Q. M., Assumpção, R. P., Padoin, A. V., Sussembach, S. P., Silva, E. N., \& Mottin, C. C. (2017). Estimated costs of clinical and surgical treatment of severe obesity in the Brazilian public health system. Obesity surgery, 27(12), 3273-3280. 